\title{
Ground state solutions for a Schrödinger-Poisson-Slater type with critical growth
}

JUN LEI ${ }^{1}$, Jiafeng Zhang ${ }^{1}$, and Chunyu Lei ${ }^{1}$

${ }^{1}$ Guizhou Minzu University

July 12, 2021

\begin{abstract}
We study the existence of ground state solutions of a Schrödinger-Poisson-Slater type equation with critical growth. By using the Nehari-Pohozaev manifold, we obtain the existence of ground state solutions of this system.
\end{abstract}

\section{Hosted file}

doub.pdf available at https://authorea.com/users/425226/articles/530093-ground-statesolutions-for-a-schr\%C3\%B6dinger-poisson-slater-type-with-critical-growth 